Journal of Animal and Veterinary Advances 10 (9): 1194-1201, 2011

ISSN: $1680-5593$

(C) Medwell Journals, 2011

\title{
Determination of Pollution Level of Yuvarlak Stream (Koycegiz-Mugla) by Using Benthic Macro Invertebrates
}

\author{
E. Tarik Topkara, Murat Ozbek, Ayse Tasdemir, Seray Yildiz, \\ Suleyman Balik and M. Rusen Ustaoglu \\ Department of Hydrobiology, Faculty of Fisheries, Ege University, \\ 35100 Bornova-Izmir, Turkey
}

\begin{abstract}
In order to determine the pollution level of Yuvarlak stream by using benthic macro invertebrates, monthly samplings were conducted between May 2001 and April 2002 at ten stations. Benthic macro invertebrates were sampled by sieving of mud samples collected by using a $500 \mu \mathrm{m}$ mesh sized kick-net and an Ekman-Birge grab (at ninth and tenth stations) through a $500 \mu \mathrm{m}$ mesh sized sieve. Collected materials were fixed in $4 \%$ formalin solution in field.
\end{abstract}

Key words: Pollution, benthic macro invertebrates, biotic index, Yuvarlak stream, formalin, sampling

\section{INTRODUCTION}

Yuvarlak stream which is one of the most important rivers joining to Koycegiz lake is both economical and ecological significance. Apart from touristic activities, the fact that it hosts to the biggest trout farm in the country is another indicator of its economical significance.

Irregular flow regime which is observed in most of the rivers in the country is not observed in Yuvarlak stream. Ecological diversity is remarkable because of continuous water flow during annual period. Drainage area of the river is under anthropogenic influence from settlement and industrial plants located in its basin.

In recent years, use of benthic invertebrate for determining pollution in river systems is a preferred method because a number of indicator taxa exist among benthic invertebrates abundant in the river systems. Additionally, some indices used enable complete explanation for obtained data.

It is remarkable that the very first studies on the subject in Yuvarlak stream were reported by Kazanci and Dugel (2000) and Barlas. Both studies aimed to determine status of pollution in the rivers using benthic macro-invertebrate. Ozbek et al. (2003) reported that the second location on which Echinogammarus antalyae (Amphipoda-Crustacea) was found in the country was Yuvarlak stream.

Ustaoglu investigated Mollusca fauna of Yuvarlak stream and reported a total of 16 taxa. Similarly, Ozbek et al. (2003) investigated Malacostraca (CrustaceaArthropoda) fauna in Yuvarlak stream and reported 11 taxa from the lake. Aygen et al. (2004) reported 2 new Ostracod (Humphcypris subterranea and Herpetocypris brevicaudata) species (Crustacea-Arthropoda) for
Turkish fauna. It was attempted to detect status of pollution in Yuvarlak stream by qualitative and quantitative samplings performed monthly within in 1 year period. It was also attempted to obtain information on change in the river system by comparing obtained data with those from the previous studies.

\section{MATERIALS AND METHODS}

Benthic macro-invertebrate were sampled monthly on a total of 10 stations chosen from the river and estuary of Yuvarlak stream between May 2001 and April 2002 (Fig. 1). Stations 5 and 6 dried out and no sampling could be performed from these stations because of water collection and irrigational activities in Beyobasi Village

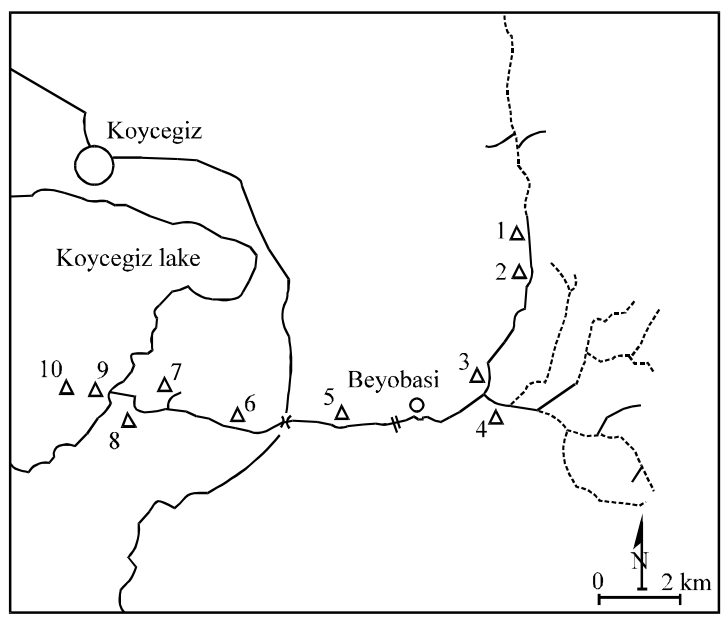

Fig. 1: Samples of benthic macro-invertebrate between May 2001-April 2002

Corresponding Author: E. Tarik Topkara, Department of Hydrobiology, Faculty of Fisheries, Ege University, 35100 Bornova-Izmir, Turkey 
between June and October 2001 during the study period. Stations 7 and 8 located on lower parts were fed by underground water and water from Nasifdede river and showed no drought. Additionally, no sampling could be performed from the station 4 on May 2001. Benthic samples were collected Ekman Grab (Stations 9 and 10) and kick-net with pore of $500 \mu \mathrm{m}$. For the samplings with Kick-net, benthic invertebrates were collected in such a way that several samples would be taken from each station.

Ekman Grab samples were then filtered through fine sieve with pore of $500 \mu \mathrm{m}$. Biological samples fixed in $4 \%$ formaldehyde at the study area were stored in $70 \%$ alcohol following washing with abundant water in the laboratory.

For identification of these materials classified according to systematic groups; studies of Bohmig were utilized for Tricladida; those of Bilgin, Zhadin(1952), Gloer for Mollusca; those of Brinkhurst and Jamieson (1971), Sperber (1950), Kathman and Brinkhurst (1998), Nielsen and Christensen (1959), Timm (1999) for Annelida; those of Viets for Acariformes; those of Bronstein, Henderson (1990), Gulen, Mozo et al. (1996), Martens (1997) for Ostracoda; those of Ariani and Wittmann (2000), Brandis et al. (2000), Holthius (1961), Karaman and Pinkster (1987), Kocatas for Malacostraca; those of Elliot et al. (1988) and Tanatmis for Ephemeroptera; those of Ris for Odonata; those of Hynes (1993) for Plecoptera; those of Savage (1989) for Heteroptera; those of Bertrand, Reitter for Coleoptera; those of Edington and Hildrew (1995) for Trichoptera; those of Sahin, Fittkau and Roback (1983), Epler (1995), Cranston (1982), Pennak (1953) for Diptera.

Benthic material obtained from samplings was assessed qualitatively and quantitatively. Shannon's Diversity Index and frequency and similarity indices were used in quantitative analyses (Kazanci and Dugel, 2000). Biotic Index of Belgium (De Pauw and Vanhooren, 1983) was preferred for determination of water quality using benthic invertebrates.

Shannon's diversity index: This index was used in calculation of species diversity of benthic invertebrates in Yuvarlak Stream. The equation is as follows:

$$
\mathrm{H}^{\prime}=\sum_{\mathrm{i}=1}^{\mathrm{s}} \mathrm{Pi} \log _{2} \mathrm{Pi}
$$

Where:

$$
\begin{aligned}
& \mathrm{H}^{\prime}=\text { Shannon's Diversity Index } \\
& \mathrm{Pi}=\mathrm{Ni} / \mathrm{N}
\end{aligned}
$$

$$
\mathrm{Ni}=\text { Number of individuals of each taxon }
$$$$
\mathrm{N}=\text { Total number of individuals }
$$

Results range between 0 and 5 in Shannon's Index when they are calculated according to logarithm with 2 being base. Stability and balance conditions exist in the communities on the locations with index values $>2.5$. One may say that pollution in the environment increases as the index value approaches to 0 .

Jaccard's Similarity Index: Jaccard's Similarity Index was used to determine similarities between stations. Index values range between 0 and 1 for this simple index based on the principle of present or absent. In the present study, values were multiplied by 100 and similarity values were given as percentage values. The equation is as follows:

Where:

$$
S_{j}=a /(a+b+c)
$$

$\mathrm{S}_{\mathrm{j}}=$ Jaccard's Similarity Index

$\mathrm{a}=$ Number of the taxa present on both of the stations

$\mathrm{b}=$ The number of the taxa present on the first station

c $=$ Number of the taxa present on the second station

Frequency: In order to calculate the frequency values, number of the samplings in which each taxon was determined was divided into the total number of the samplings:

Where:

$$
\mathrm{F}=(\mathrm{Na} / \mathrm{N}) \times 100
$$

$\mathrm{F}=$ Frequency

$\mathrm{Na}=$ Number of the samplings containing taxon a

$\mathrm{N}=$ Total number of the samplings

\section{RESULTS AND DISCUSSION}

At the end of benthic samplings from Yuvarlak stream, a total of 46.907 individuals were detected of 125 species belonging to Tricladida, Mollusca (Gastropoda, Bivalvia), Annelida (Oligochaeta, Hirudinea), Acariformes, Ostracoda, Mysidacea, Amphipoda, Decapoda, Isopoda, Ephemeroptera, Odonata, Plecoptera, Heteroptera, Coleoptera, Trichoptera and Diptera groups.

During the sampling period, the highest number of the individuals were observed on October 2001 and the lowest number of the individuals was observed on December 2001 (Fig. 2).

Highest number of individuals during the study period was observed on the third station. Amount of the individuals sampled from this particular station makes $41.25 \%$ of the total number of the individuals with the lowest number of individuals having observed on the station 6 (Fig. 3). This is due to the fact that the stations 5 and 6 were dry out between June and October 2001 during the sampling period not to pollution emerging in 


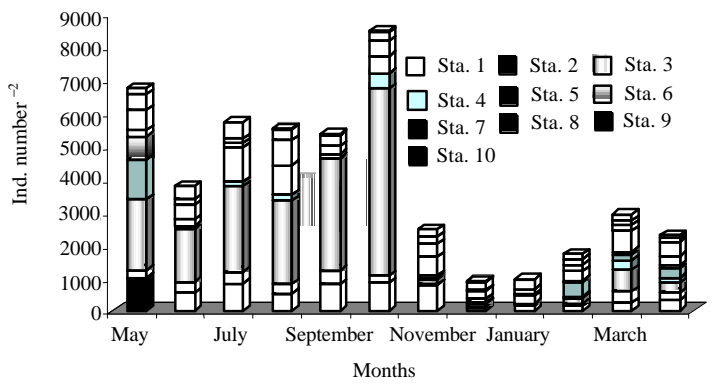

Fig. 2: The highest number of indivisuals observed on October 2001 and the lowest number of indivisuals observed on December 2001

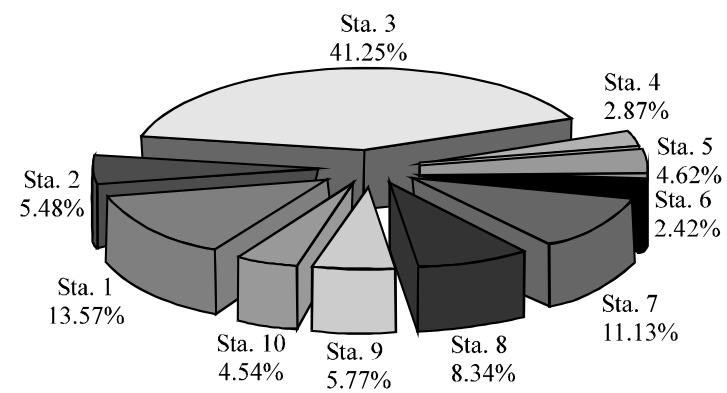

Fig. 3: Highest number of indivisuals observed on station 3

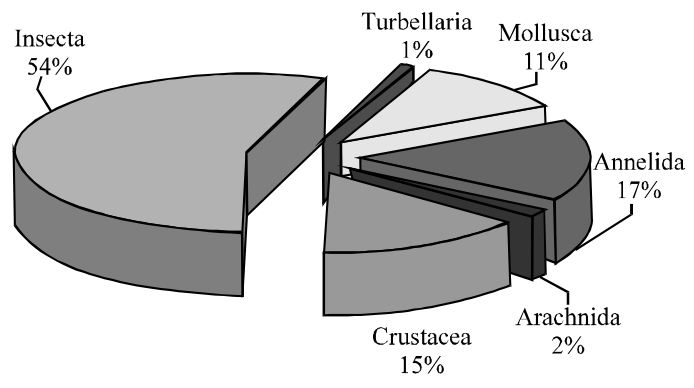

Fig. 4: Insecta with regard to the number of species made the biggest group

these stations. In regard to the numbers of the species belonging to the groups detected, the biggest group is made by Insecta with a rate of $54 \%$ followed by Annelida $(17 \%)$ and Crustacea (15\%) (Fig. 4). Systematic classifications of the taxa belonging to these groups were given according to Parker (1982) (Table 1) and distribution of the taxa by the stations was shown in Table 1 .

Perla, Agapetus, Hydroptila, Dixa, Ilyocypris were found only in the station 1 whereas Orecthochilus, Diplectrona, Cheumatopsyche, Wormaldia was found in the station 2; Ophiogomphus, Chimarra in the station 3; Torrenticola in the station 4; Limnophora in the station 5; Psychomyiidae, Palpomyia in the station 6; Peltodytes, Cyrnus, Metalype, Hydroptilidae, Limnephilidae in the
Table 1: Systematic classification and distribution of the texa

\begin{tabular}{|c|c|c|c|c|c|c|c|c|c|c|}
\hline Taxa & 1 & 2 & 3 & 4 & 5 & 6 & 7 & 8 & 9 & 10 \\
\hline Dugesia & * & * & $*$ & $*$ & $*$ & $*$ & $*$ & $*$ & - & - \\
\hline Theodoxus & $*$ & $*$ & - & - & - & - & * & $*$ & $*$ & * \\
\hline Potamopyrgus & - & - & - & - & - & - & - & - & $*$ & - \\
\hline Melanopsis & - & - & - & - & - & - & $*$ & $*$ & - & - \\
\hline Melanoides & - & - & - & - & - & - & & $*$ & $*$ & * \\
\hline Bithynia & - & - & - & - & - & - & $*$ & $*$ & - & - \\
\hline Valvata & - & - & - & - & * & $*$ & * & $*$ & * & \\
\hline Radix & - & - & $*$ & - & $*$ & $*$ & $*$ & $*$ & - & $*$ \\
\hline Ancylus & * & $*$ & $*$ & - & - & $*$ & - & $*$ & - & - \\
\hline Physella & - & - & $*$ & - & $*$ & $*$ & $*$ & $*$ & - & - \\
\hline Gyraulus & - & - & $*$ & - & $*$ & $*$ & $*$ & * & * & - \\
\hline Planorbarius & - & - & - & - & - & - & $*$ & $*$ & - & - \\
\hline Planorbis & - & - & $*$ & - & - & $*$ & * & - & - & - \\
\hline Succinea & - & - & - & - & - & - & $*$ & - & - & - \\
\hline Pisidium & $*$ & $*$ & $*$ & - & $*$ & $*$ & $*$ & $*$ & - & - \\
\hline Albaglossiphonia & - & - & - & - & - & - & - & * & - & - \\
\hline Haementeria & - & - & - & - & $*$ & - & - & $*$ & - & - \\
\hline Erpobdella & - & - & $*$ & - & $*$ & $*$ & - & $*$ & - & - \\
\hline Tubificidae & $*$ & $*$ & $*$ & $*$ & $*$ & $*$ & $*$ & $*$ & * & $*$ \\
\hline Naididae & $*$ & - & $*$ & $*$ & $*$ & $*$ & $*$ & $*$ & $*$ & $*$ \\
\hline Enchy traeidae & $*$ & - & $*$ & - & $*$ & $*$ & $*$ & $*$ & $*$ & $*$ \\
\hline Lumbriculidae & - & - & $*$ & - & - & - & * & $*$ & - & - \\
\hline Lumbricidae & $*$ & $*$ & $*$ & - & - & $*$ & - & $*$ & - & - \\
\hline Atractides & $*$ & - & - & * & - & - & - & - & - & - \\
\hline Torrentic ola & - & - & - & * & - & - & - & - & - & - \\
\hline Ilyocypris & $*$ & - & - & - & - & - & - & - & - & - \\
\hline Candona & - & - & $*$ & - & - & * & - & - & - & - \\
\hline Heterocypris & * & - & - & - & - & - & - & $*$ & - & - \\
\hline Herpetocypris & - & - & $*$ & $*$ & $*$ & $*$ & - & * & - & - \\
\hline Psychrodromus & * & - & $*$ & $*$ & $*$ & $*$ & - & $*$ & - & - \\
\hline Prionocypris & - & - & - & - & - & - & - & $*$ & - & - \\
\hline Humpcypris & $*$ & - & - & * & - & - & - & - & - & - \\
\hline Eucypris & * & - & - & - & - & $*$ & - & - & - & - \\
\hline Potamocypris & - & - & - & - & - & - & - & $*$ & - & - \\
\hline Diamysis & - & - & - & - & - & - & - & - & $*$ & - \\
\hline Palaemonetes & - & - & - & - & - & - & $*$ & $*$ & * & - \\
\hline Aty aepyra & - & - & - & - & - & - & - & * & - & $*$ \\
\hline Potamon & - & $*$ & - & $*$ & - & - & $*$ & * & - & - \\
\hline Gammarus & $*$ & $*$ & $*$ & - & - & - & - & - & - & - \\
\hline Echinogammarus & - & - & - & - & - & - & * & * & $*$ & $*$ \\
\hline Corophium & - & - & - & - & - & - & - & - & $*$ & $*$ \\
\hline Orchestia & - & - & - & - & - & - & - & - & $*$ & \\
\hline Lekanesphaera & - & - & - & - & - & - & * & - & $*$ & $*$ \\
\hline Cyathura & - & - & - & - & - & - & - & - & - & * \\
\hline Baetis & $*$ & $*$ & $*$ & $*$ & $*$ & $*$ & $*$ & $*$ & $*$ & - \\
\hline Rhitrogenia & $*$ & $*$ & $*$ & - & - & $*$ & - & - & - & - \\
\hline Ephemerella & $*$ & $*$ & $*$ & - & $*$ & * & $*$ & $*$ & - & - \\
\hline Caenis & - & $*$ & $*$ & - & $*$ & $*$ & $*$ & $*$ & $*$ & - \\
\hline Ephemera & - & - & - & - & - & - & * & $*$ & $*$ & - \\
\hline Cloeon & - & - & - & - & - & - & * & $*$ & - & - \\
\hline Calopteryx & - & - & - & - & - & - & $*$ & $*$ & - & - \\
\hline Agrion & - & - & - & - & - & - & - & * & - & - \\
\hline Platycnemis & - & - & - & - & - & - & $*$ & $*$ & - & - \\
\hline Anax & $*$ & $*$ & $*$ & $*$ & - & - & - & - & - & - \\
\hline Gomphus & - & - & - & * & $*$ & $*$ & * & $*$ & - & - \\
\hline Ony chogomphus & - & $*$ & $*$ & * & $*$ & $*$ & - & $*$ & - & - \\
\hline Ophiogomphus & - & - & $*$ & - & - & - & - & $*$ & - & - \\
\hline Cordulegaster & $*$ & $*$ & $*$ & - & - & - & - & - & - & - \\
\hline Orthetrum & - & - & - & * & - & - & - & $*$ & - & - \\
\hline Coenagrion & - & - & - & - & - & - & * & $*$ & $*$ & $*$ \\
\hline Protonemura & $*$ & $*$ & - & - & - & - & - & - & - & - \\
\hline Leuctra & - & $*$ & - & - & - & - & - & - & - & - \\
\hline Perla & $*$ & - & - & - & - & - & - & - & - & - \\
\hline Sigara & - & - & - & - & $*$ & * & * & $*$ & - & - \\
\hline Gerris & - & - & - & - & $*$ & - & - & $*$ & - & - \\
\hline Aphelocheirus & - & - & - & - & - & - & $*$ & $*$ & - & - \\
\hline Microvelia & - & - & - & - & - & - & - & $*$ & - & - \\
\hline Gyrinus & - & $*$ & - & - & - & - & - & $*$ & - & - \\
\hline Laccophilus & - & - & - & - & - & - & - & $*$ & - & - \\
\hline Peltodytes & - & - & - & - & - & - & $*$ & - & - & \\
\hline Elmidae & * & $*$ & * & * & $*$ & $*$ & * & $*$ & - & - \\
\hline
\end{tabular}




\begin{tabular}{|c|c|c|c|c|c|c|c|c|c|c|}
\hline$\underline{\text { Taxa }}$ & 1 & 2 & 3 & 4 & 5 & 6 & 7 & 8 & 9 & 10 \\
\hline Donacia & - & - & - & - & - & - & - & $*$ & - & - \\
\hline Orechtochilus & - & $*$ & - & - & - & - & - & - & - & - \\
\hline Dytiscidae & - & - & - & - & - & $*$ & $*$ & * & - & - \\
\hline Rhycophila & * & * & $*$ & $*$ & $*$ & * & & & & \\
\hline Glossosoma & * & $*$ & $*$ & - & $*$ & * & $*$ & * & - & - \\
\hline Agapetus & * & - & - & - & - & - & - & - & - & - \\
\hline Hydropsyche & * & * & $*$ & - & $*$ & * & * & * & & \\
\hline Diplectrona & - & * & - & - & - & - & - & - & - & - \\
\hline Cheumatopsyche & - & $*$ & - & - & - & - & - & - & - & - \\
\hline Polycentropus & - & $*$ & - & * & - & $*$ & $*$ & * & - & - \\
\hline Cyrnus & - & - & - & - & - & - & * & - & - & - \\
\hline Psychomyiidae & - & - & - & - & - & * & - & - & - & - \\
\hline Metalype & - & - & - & - & - & - & * & - & - & - \\
\hline Hydroptilidae & - & - & - & - & - & - & $*$ & - & - & - \\
\hline Hydroptila & * & - & - & - & - & - & - & - & - & - \\
\hline Chimarra & - & - & * & - & - & - & - & - & - & - \\
\hline Wormaldia & - & $*$ & - & - & - & - & - & - & - & - \\
\hline Leptoceridae & * & * & $*$ & - & - & * & - & * & - & - \\
\hline Limnephilidae & - & - & - & - & - & - & * & - & - & - \\
\hline Tipula & * & * & & * & $*$ & - & - & * & - & - \\
\hline Limonia & * & - & $*$ & - & - & - & - & - & - & - \\
\hline Dicranota & * & - & - & - & - & - & - & * & - & - \\
\hline Dixa & * & - & - & - & - & - & - & - & - & - \\
\hline Culicoides & - & - & - & - & - & - & - & * & - & - \\
\hline Palpomyia & - & - & - & - & - & $*$ & - & - & - & - \\
\hline Liponeura & - & $*$ & - & - & - & - & - & * & - & - \\
\hline Bibiocephala & * & $*$ & - & * & - & - & - & * & * & - \\
\hline Chrysops & * & - & $*$ & - & $*$ & - & - & $*$ & - & - \\
\hline Haemotopota & - & - & - & 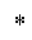 & - & - & * & $*$ & - & - \\
\hline Tabanus & * & - & - & - & - & - & $*$ & - & - & - \\
\hline Atherix & * & $*$ & $*$ & - & $*$ & - & $*$ & - & $*$ & - \\
\hline Limnophora & - & - & - & - & * & - & - & - & - & - \\
\hline Empididae & - & - & - & - & . & * & - & * & $*$ & - \\
\hline Simuliidae & * & * & * & * & * & * & * & $*$ & - & - \\
\hline Chironomidae & $*$ & * & $*$ & $*$ & * & $*$ & * & $*$ & * & $*$ \\
\hline
\end{tabular}

station 7; Albaglossiphonia, Prionocypris, Potamocypris, Agrion, Donacia, Microvelia, Laccophilus, Culicoides in the Station 8; Potamopyrgus, Diamysis and Orchestia in the station 9 and Cyathura in the station 10 .

Frequency values of the benthic invertebrates detected according to the stations were shown in Table 2. Accordingly, frequency values of Theodoxus, Gammarus and Baetes species in the first station was found to be $100 \%$. Frequeuncy values of the Gammarus and Baetis species reached to $100 \%$ in the second station as in the first station whereas frequency value of Hydropsyche reached to $100 \%$ only in this station. It is remarkable that frequency values of Dugesia, Gyraulus, Tubificidae and Chrinomidae is $100 \%$ in the station 3 . No taxon was found to reach frequency value of $100 \%$ in the stations 4,5 and 6 (because of drought occurred on the 5th and 6th months) whereas Gyraulus in the station 7, Echinogammarus in the station 8 and Echinogammarus in the station 9 reached frequency values of $100 \%$. It is also remarkable that there was no taxon reaching to this frequency value in the station 10 (Table 2). Additionally, it was found that the individuals of Chironomidae species found in all stations reached a frequency value of $78.3 \%$ and similarly; mean frequency value of Tubificidae members was $53.3 \%$.

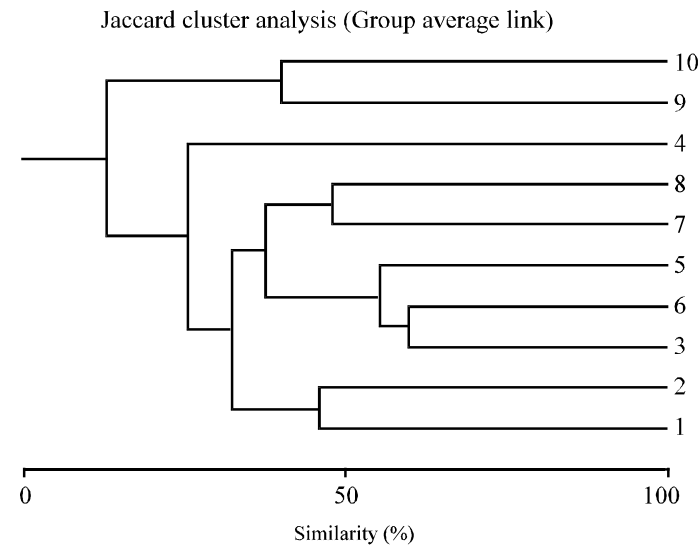

Fig. 5: The brackish quantity and fresh air quantity stations

Values of diversity index calculated based on 12 month sampling from Yuvarlak stream was shown in the Table 3. Accordingly, the highest diversity values were seen on March in the stations 1 and 2 on May in the stations 3,56 and 8; on April in the station 4; on August and February in the station 7, on March in the station 9 and on April in the station 10. Based on samplings from Yuvarlak stream and calculated similarity index, the highest index value was seen in the stations 3 through 6 $(60 \%)$ with the station 5 accompanying these 2 stations. Taken together, the stations 9 and 10 seem to be quite different from others although they are relatively similar to each other. The main reason for this is that these 2 stations are of brackish quality whereas others are of fresh water quality (Fig. 5).

Biotic Index of Belgium was used in calculations of biotic index. The highest and the lowest biotic index in the station 1 were found to be 10 and 4, respectively. Biotic index value reached to 10 on May in this station which exhibited biotic indices close to 7-8 in months other than November and December. With these results, the station 1 belongs to the group of un-polluted or slightly polluted waters (Table 4). This station seems to be cleaner than others because it locates on the source region. Degree of pollution in the station 2 increased slightly although values of biotic index range between 6 and 8 in this station. Thus, it may be included in the group of slightly polluted water. A decrease was seen in the station 3 with a mean index value being 6 . Thus, this station gained a critical position and was included in the category of relatively polluted waters. Among the stations studied, the lowest value of biotic index was found in the station 4 with an index value being 4 . With this value, station 4 is included in the category of polluted waters. Mean values of biotic index was calculated to be 5 in the stations 5 and 6 where no sampling was performed due to drought for a period of 5 months. In these stations, index value of 1 calculated for month January is an exceptional situation. 
J. Anim. Vet. Adv., 10 (9): 1194-1201, 2011

Table 2: Frequency values of the benthic invertebrates detected according to the station

\begin{tabular}{|c|c|c|c|c|c|c|c|c|c|c|}
\hline Taxa & 1 & 2 & 3 & 4 & 5 & 6 & 7 & 8 & 9 & 10 \\
\hline Dugesia & 91.7 & 91.7 & 100.0 & 50.0 & 33.3 & 33.3 & 50.0 & 16.7 & - & - \\
\hline Theodoxus & 100.0 & 41.7 & - & - & - & - & 75.0 & 58.3 & 50.0 & 25.0 \\
\hline Potamopyrgus & - & - & - & - & - & - & - & - & 16.7 & - \\
\hline Melanopsis & - & - & - & - & - & - & 75.0 & 75.0 & - & - \\
\hline Melanoides & - & - & - & - & - & - & - & 8.3 & 25.0 & 16.7 \\
\hline Bithynia & - & - & - & - & - & - & 91.7 & 16.7 & - & - \\
\hline Valvata & - & - & - & - & 25.0 & 33.3 & 83.3 & 83.3 & - & - \\
\hline Radix & - & - & 66.7 & - & 25.0 & 41.7 & 75.0 & 25.0 & - & 8.3 \\
\hline Ancylus & 25.0 & 8.3 & 91.7 & - & - & 8.3 & - & 8.3 & - & - \\
\hline Physella & - & - & 75.0 & - & 8.3 & 8.3 & 25.0 & 50.0 & - & - \\
\hline Gyraulus & - & - & 100.0 & - & 25.0 & 25.0 & 100.0 & 58.3 & 8.3 & - \\
\hline Planorbarius & - & - & - & - & - & - & 16.7 & - & - & - \\
\hline Planorbis & - & - & 8.3 & - & - & 8.3 & 25.0 & - & - & - \\
\hline Succinea & - & - & - & - & - & - & 8.3 & - & - & - \\
\hline Pisidium & 8.3 & 8.3 & 58.3 & - & 25.0 & 16.7 & 75.0 & 50.0 & - & - \\
\hline Albaglossiphonia & - & - & - & - & - & - & - & 8.3 & - & - \\
\hline Haementeria & - & - & - & - & 8.3 & - & - & 8.3 & - & - \\
\hline Erpobdella & - & - & 33.3 & - & 8.3 & 16.7 & - & 8.3 & - & - \\
\hline Tubificidae & 8.3 & 8.3 & 100.0 & 25.0 & 33.3 & 16.7 & 83.3 & 91.7 & 100.0 & 66.7 \\
\hline Naididae & 8.3 & - & 50.0 & 25.0 & 25.0 & 25.0 & 25.0 & 41.7 & 25.0 & 33.3 \\
\hline Enchytraeidae & 8.3 & - & 25.0 & - & 16.7 & 25.0 & 8.3 & 33.3 & 8.3 & 8.3 \\
\hline Lumbriculidae & - & - & 8.3 & - & - & - & 8.3 & 8.3 & - & - \\
\hline Lumbricidae & 16.7 & 25.0 & 8.3 & - & - & 8.3 & - & 8.3 & - & - \\
\hline Atractides & 8.3 & - & - & 8.3 & - & - & - & - & - & - \\
\hline Torrenticola & - & - & - & 8.3 & - & - & - & - & - & - \\
\hline Ilyocypris & 8.3 & - & - & - & - & - & - & - & - & - \\
\hline Candona & - & - & 8.3 & - & - & 8.3 & - & - & - & - \\
\hline Heterocypris & 8.3 & - & - & - & - & - & - & 8.3 & - & - \\
\hline Herpetocypris & - & - & 50.0 & 25.0 & 8.3 & 16.7 & - & 8.3 & - & - \\
\hline Psychrodromus & 25.0 & - & 58.3 & 8.3 & 25.0 & 25.0 & - & 16.7 & - & - \\
\hline Prionocypris & - & - & - & - & - & - & - & 8.3 & - & - \\
\hline Humpcypris & 8.3 & - & - & 8.3 & - & - & - & - & - & - \\
\hline Eucypris & 25.0 & - & - & - & - & 8.3 & - & - & - & - \\
\hline Potamocypris & - & - & - & - & - & - & - & 8.3 & - & - \\
\hline Diamysis & - & - & - & - & - & - & - & - & 8.3 & - \\
\hline Palaemonetes & - & - & - & - & - & - & 66.7 & 25.0 & 8.3 & 8.3 \\
\hline Atyaepyra & - & - & - & - & - & - & - & 8.3 & - & - \\
\hline Potamon & - & 8.3 & - & 25.0 & - & - & 58.3 & 8.3 & - & - \\
\hline Gammarus & 100.0 & 100.0 & 16.7 & - & - & - & - & - & - & - \\
\hline Echinogammarus & - & - & - & - & - & - & 75.0 & 100.0 & 16.7 & 33.3 \\
\hline Corophium & - & - & - & - & - & - & - & - & 83.3 & 75.0 \\
\hline Orchestia & - & - & - & - & - & - & - & - & 8.3 & - \\
\hline Lekanesphaera & - & - & - & - & - & - & 8.3 & - & 25.0 & 16.7 \\
\hline Cyathura & - & - & - & - & - & - & - & - & - & 8.3 \\
\hline Baetis & 100.0 & 100.0 & 83.3 & 41.7 & 50.0 & 33.3 & 66.7 & 50.0 & 16.7 & - \\
\hline Rhitrogenia & 25.0 & 66.7 & 25.0 & - & - & 8.3 & - & - & - & - \\
\hline Ephemerella & 16.7 & 75.0 & 25.0 & - & 25.0 & 16.7 & 16.7 & 16.7 & - & - \\
\hline Caenis & - & 8.3 & 33.3 & 50.0 & - & 25.0 & 33.3 & 50.0 & 25.0 & - \\
\hline Ephemera & - & - & 8.3 & 8.3 & 16.7 & 8.3 & 8.3 & 16.7 & 8.3 & - \\
\hline Cloeon & - & - & - & - & - & - & 8.3 & 8.3 & - & - \\
\hline Calopteryx & - & - & - & - & - & - & 33.3 & 16.7 & - & - \\
\hline Agrion & - & - & - & - & - & - & - & 8.3 & - & - \\
\hline Platycnemis & - & - & - & - & - & - & 8.3 & 8.3 & - & - \\
\hline Anax & 41.7 & 75.0 & 8.3 & 25.0 & - & - & - & 16.7 & - & - \\
\hline Gomphus & - & - & - & 16.7 & 8.3 & 8.3 & 25.0 & 16.7 & - & - \\
\hline Ony chogomphus & - & 8.3 & 33.3 & 16.7 & 25.0 & 8.3 & - & 16.7 & - & - \\
\hline Ophiogomphus & - & 8.3 & 8.3 & - & - & - & - & - & - & - \\
\hline Cordulegaster & 8.3 & 8.3 & 16.7 & - & - & - & - & - & - & - \\
\hline Orthetrum & - & - & - & 8.3 & - & - & - & 16.7 & - & - \\
\hline Coenagrion & - & - & - & - & - & - & 8.3 & 8.3 & 8.3 & 8.3 \\
\hline Protonemura & 83.3 & 50.0 & - & - & - & - & - & - & - & - \\
\hline Leuctra & - & 8.3 & - & - & - & - & - & - & - & - \\
\hline Perla & 33.3 & - & - & - & - & - & - & - & - & - \\
\hline Sigara & - & - & - & - & 8.3 & 8.3 & 25.0 & 16.7 & - & - \\
\hline Gerris & - & - & - & - & 8.3 & - & - & 8.3 & - & - \\
\hline Aphelocheirus & - & - & - & - & - & - & 83.3 & 16.7 & - & - \\
\hline Microvelia & - & - & - & - & - & - & - & 8.3 & - & - \\
\hline Gyrinus & - & 8.3 & - & - & - & - & - & 8.3 & - & - \\
\hline Laccophilus & - & - & - & - & - & - & - & 8.3 & - & - \\
\hline
\end{tabular}




\begin{tabular}{|c|c|c|c|c|c|c|c|c|c|c|}
\hline Taxa & 1 & 2 & 3 & 4 & 5 & 6 & 7 & 8 & 9 & 10 \\
\hline Peltodytes & - & - & - & - & - & - & 8.3 & - & - & - \\
\hline Elmidae & 75.0 & 83.3 & 16.7 & 25.0 & 8.3 & 25.0 & 41.7 & 8.3 & - & - \\
\hline Donacia & - & - & - & - & - & - & - & 8.3 & - & - \\
\hline Orechtochilus & - & 8.3 & - & - & - & - & - & - & - & - \\
\hline Dytiscidae & - & - & - & - & - & 8.3 & 8.3 & 8.3 & - & - \\
\hline Rhycophila & 50.0 & 58.3 & 8.3 & 8.3 & 8.3 & 8.3 & - & - & - & - \\
\hline Glossosoma & 58.3 & 58.3 & 16.7 & - & 25.0 & 16.7 & 8.3 & 8.3 & - & - \\
\hline Agapetus & 16.7 & - & - & - & - & - & - & - & - & - \\
\hline Hydropsyche & 41.7 & 100.0 & 33.3 & - & 16.7 & 16.7 & 33.3 & - & - & - \\
\hline Diplectrona & - & 16.7 & 8.3 & - & - & - & - & - & - & - \\
\hline Cheumatopsyche & - & 16.7 & - & - & - & 8.3 & 8.3 & - & - & - \\
\hline Polycentropus & - & 8.3 & - & 8.3 & - & 8.3 & 41.7 & 8.3 & - & - \\
\hline Cyrnus & - & - & - & - & - & - & 8.3 & - & - & - \\
\hline Psychomyiidae & - & - & - & - & - & 8.3 & - & - & - & - \\
\hline Metalype & - & - & - & - & - & - & 8.3 & - & - & - \\
\hline Hydroptilidae & - & - & - & - & & - & 8.3 & - & - & - \\
\hline Hydroptila & 8.3 & - & - & 8.3 & - & - & - & - & - & - \\
\hline Chimarra & - & - & 8.3 & - & - & - & - & - & - & - \\
\hline Wormaldia & - & 8.3 & - & - & - & - & - & - & - & - \\
\hline Leptoceridae & 25.0 & 41.7 & 16.7 & - & - & 8.3 & - & 8.3 & - & - \\
\hline Limnephilidae & - & - & - & - & - & - & 8.3 & - & - & - \\
\hline Tipula & 16.7 & - & 8.3 & 8.3 & 8.3 & - & - & 8.3 & - & - \\
\hline Limonia & 16.7 & - & 8.3 & - & - & - & - & - & - & - \\
\hline Dicranota & 8.3 & - & - & - & - & - & - & 8.3 & - & - \\
\hline Dixa & 8.3 & - & 16.7 & - & 8.3 & 8.3 & 16.7 & 8.3 & - & - \\
\hline Culicoides & - & - & - & - & - & - & - & 8.3 & - & - \\
\hline Palpomyia & - & - & - & - & - & - & 8.3 & - & - & - \\
\hline Liponeura & - & - & 8.3 & - & - & - & - & 8.3 & - & - \\
\hline Bibiocephala & 16.7 & 16.7 & - & 8.3 & - & - & - & 8.3 & 8.3 & - \\
\hline Chrysops & 8.3 & - & 8.3 & - & 8.3 & - & - & - & 8.3 & - \\
\hline Haemotopota & - & - & - & 8.3 & - & - & 8.3 & 8.3 & - & - \\
\hline Tabanus & 8.3 & - & - & - & - & - & 16.7 & - & - & - \\
\hline Atherix & 25.0 & 33.3 & 25.0 & - & 8.3 & - & 8.3 & - & 8.3 & - \\
\hline Limnophora & - & - & 8.3 & - & - & - & - & - & - & - \\
\hline Empididae & - & - & - & - & - & 8.3 & - & 8.3 & 8.3 & - \\
\hline Simuliidae & 8.3 & 66.7 & 75.0 & 50.0 & 41.7 & 58.3 & 41.7 & 33.3 & - & - \\
\hline Chironomidae & 83.3 & 66.7 & 100.0 & 66.7 & 58.3 & 58.3 & 83.3 & 91.7 & 91.7 & 83.3 \\
\hline
\end{tabular}

Table 3: Values of the diversity index calculated based on 12 month sampling from Yuvarlak stream

\begin{tabular}{|c|c|c|c|c|c|c|c|c|c|c|c|c|}
\hline St. & May & Jun. & Jul. & Aug. & Sep. & Oct. & Nov. & Dec. & Jan. & Feb. & Mar. & Apr. \\
\hline 1 & 4.087 & 3.459 & 3.322 & 3.585 & 3.807 & 1.984 & 3.807 & 2.322 & 2.279 & 3.322 & 4.644 & 2.585 \\
\hline 2 & 3.907 & 3.459 & 3.700 & 3.459 & 4.000 & 3.295 & 3.170 & 3.322 & 2.683 & 3.585 & 4.170 & 4.000 \\
\hline 3 & 4.524 & 4.322 & 3.807 & 3.700 & 3.907 & 2.176 & 3.700 & 3.322 & 2.196 & 3.459 & 4.322 & 4.087 \\
\hline 4 & - & 2.000 & 2.000 & 3.322 & 1.585 & 1.251 & 2.000 & 1.000 & 0.000 & 2.322 & 3.459 & 3.700 \\
\hline 5 & 4.322 & - & - & - & - & - & 3.170 & 2.322 & 1.278 & 4.170 & 2.322 & 3.459 \\
\hline 6 & 4.459 & - & - & - & - & - & 3.170 & 3.322 & 0.919 & 3.807 & 3.459 & 3.700 \\
\hline 7 & 4.000 & 3.459 & 4.248 & 4.524 & 3.907 & 2.095 & 4.000 & 3.700 & 2.337 & 4.524 & 3.807 & 4.392 \\
\hline 8 & 4.585 & 3.585 & 3.459 & 4.322 & 3.700 & 1.857 & 3.459 & 3.170 & 2.323 & 3.907 & 3.907 & 4.322 \\
\hline 9 & 2.322 & 1.585 & 2.000 & 2.000 & 2.585 & 0.988 & 2.585 & 3.170 & 2.529 & 1.585 & 3.459 & 2.000 \\
\hline 10 & 2.000 & 2.000 & 1.585 & 1.585 & 2.000 & 0.000 & 2.000 & 1.585 & 1.513 & 2.585 & 2.000 & 2.585 \\
\hline
\end{tabular}

Table 4: The station 1 belongs to the group of un-polutedor slightly polluted waters

\begin{tabular}{|c|c|c|c|c|c|c|c|c|c|c|c|c|c|c|}
\hline St. & May & Jun. & Jul. & Aug. & Sep. & Oct. & Nov. & Dec. & Jan. & Feb. & Mar. & Apr. & Min.-Max. & Mean \\
\hline 1 & 10 & 7 & 7 & 7 & 7 & 8 & 6 & 4 & 7 & 8 & 8 & 7 & $4-10$ & 7 \\
\hline 2 & 7 & 8 & 8 & 7 & 8 & 9 & 6 & 5 & 6 & 7 & 8 & 8 & $5-9$ & 7 \\
\hline 3 & 8 & 7 & 6 & 6 & 6 & 6 & 5 & 5 & 4 & 5 & 9 & 8 & $4-9$ & 6 \\
\hline 4 & $*$ & 4 & 4 & 5 & 4 & 4 & 1 & 4 & 3 & 4 & 5 & 5 & $1-5$ & 4 \\
\hline 5 & 8 & $*$ & $*$ & $*$ & $*$ & $*$ & 4 & 6 & 1 & 8 & 4 & 6 & $1-8$ & 5 \\
\hline 6 & 8 & $*$ & $*$ & $*$ & $*$ & $*$ & 2 & 5 & 1 & 5 & 6 & 5 & $1-8$ & 5 \\
\hline 7 & 7 & 5 & 7 & 7 & 6 & 7 & 7 & 6 & 5 & 6 & 6 & 7 & $5-7$ & 6 \\
\hline$\underline{8}$ & 7 & 5 & 5 & 7 & 5 & 6 & 5 & 5 & 5 & 7 & 7 & 6 & $5-7$ & 6 \\
\hline
\end{tabular}

Biotic index values of the stations 7 and 8 ranged between 5 and 7 . These stations mean value of which was 6 are included in the category of relatively polluted waters, as the stations 5 and 6 . Values of biotic index couldn't be calculated for the stations 9 and 10 because they were located in the boundaries of the lake (Table 5). A total of 
Table 5: Values of diotic index located in the boundaries of the lake

\begin{tabular}{lcl} 
Class & Biotic Index & Condition \\
\hline I & $10-9$ & Unpolluted or slightly polluted \\
II & $8-7$ & Slightly polluted \\
III & $6-5$ & Moderately polluted or critic state \\
IV & $4-3$ & Polluted \\
V & $2-0$ & Heavily polluted \\
\hline
\end{tabular}

125 genera were found belonging to the groups of Tricladida, Mollusca (Gastropoda, Bivalvia), Annelida (Oligochatea, Hirudinae), Acariformes, Ostracoda, Mysidacea, Amphipoda, Decapoda, Isopoda, Ephemeroptera, Odonata, Plecoptera, Heteroptera, Coleoptera, Trichoptera and Diptera as a result of benthic sampling performed monthly on Yuvarlak stream between May 2001 and April 2002. In regard to the previous studies in the region, it is remarkable that Kazanci and Dugel (2000) reported on a total of 92 genera over the 7 sampling period between 1992 and 1993 and Barlas reported on 45 genera between 1998 and 2000 . On annual basis, one might claim that the stations 1 and 2 possess very high values of diversity index and accordingly, pollution in these stations doesn't have an advers effect on the benthic community. Although, the values as high as in the stations 1 and 2 were not seen in other stations than the stations 4,9 and 10 , no critical values were reached below which the effects of pollution began to be seen. In these 3 stations (stations 4,9 and 10) diversity indices were below 2.5 and it was concluded that an advers condition existed arising from these stations. This thesis is supported further by the observations from the field studies. The highest diversity index was found to be 2.06 in the study by Kazanci and Dugel (2000) and overall conclusion drawn from that study was that mild to moderate degree of pollution existed in all stations.

In regard to the similarity values between the stations, it was noteworthy that the stations 3 and 6 had a very high similarity rate between each other with the station 5 having close values to these 2 stations. Similarity of the stations 9 and 10 to the others was found to be low because of location of these 2 stations in the lake environment. In the previous study by Kazanci and Dugel (2000) reported that the station 8 located on the estuary of the river and Nasifdede stream (station \#6) had close values to each other with the station 3 being similar to these 2 stations. The stations with the highest similarity index were the stations 1 and 2 in the study by Barlas. Similarity rates of other 2 stations were equal to that of this group. Based on the observation performed on the field studies, one may argue that the calculated indices and drawn dendogram using Jaccard's similarity index gave quite accurate results.

In regard to the biotic index values according to the stations, it draws attention that the cleanest station was the station 1. The locality with the lowest biotic index value was found to be station 4 . It is considered that pollution increased in the station 4 as a consequence of organic and inorganic fertilizer from the fields around the river under the effects of rain. The stations from which sampling were performed in the study by Kazanci and Dugel (2000) were generally reported to be of first and second quality. It is remarkable that values of water quality were lower compared to the results of the present study. The main reason for this is that sampling was performed in the period of 1992-1993 in the study of Kazanci and Dugel. It is possible that pollution increased over a period of about 10 years since that time. Similarly, study by Barlas reported that values of water quality ranged between the first and second classes.

\section{CONCLUSION}

In determining of pollution level, Belgian Biotic Index was used and it was observed that the averages of index values changed from 4-7 during the year. In this case, Yuvarlak stream can be placed between the groups of polluted waters and slightly polluted waters.

\section{ACKNOWLEDGEMENTS}

We would want to thank to Associate Professor Dr. Hasan M. Sari, Dr. Cem Aygen and Researcher Ali Ilhan who provided assistance in the field studies and Dr. Cem Aygen who identified the taxa of Odonata and Ostracoda.

\section{REFERENCES}

Ariani, A.P. and K.J. Wittmann, 2000. Interbreeding versus morphological and ecological differentiation in Mediterranean Diamysis (Crustacea, Mysidacea), with description of four new taxa. Hydrobiologia, 441: 185-236.

Aygen, C., S. Balik and M.R. Ustaoglu, 2004. Two new records for the non-marine ostracod fauna of Turkey: Humphcypris subterranea (Hartmann, 1964) and Herpetocypris brevicaudata Kaufmann, 1900. Zool. Middle East, 31: 77-81.

Brandis, D., V. Storch and M. Turkay, 2000. Taxonomy and zoogeography of the freshwater crabs of Europe, North Africa and the Middle East. Senckenbergiana Biologica, 80: 5-56.

Brinkhurst, R.O. and B.G.M. Jamieson, 1971. Aquatic Oligocaeta of the World. University of Toronto Press, Toronto.

Cranston, P.S., 1982. A Key to The Larvae of the British Orthocladiinae (Chironomidae). Freshwater Biological Association, UK., pp: 152. 
De Pauw, N. and G. Vanhooren, 1983. Method for biological quality assessment of water courses in Belgium. Hydrobiologia, 100: 153-168.

Edington, J.M. and A.G. Hildrew, 1995. Caseless Caddis Larvae of the British Isles: A Key with Ecological Notes. Freshwater Biological Association, UK., pp: 134.

Elliot, J.M., U.H. Humpesch and T.T. Macan, 1988. Larvae of the British Ephemeroptera: A Key with Ecological Notes. Freshwater Biological Association, UK., pp: 145.

Epler, J.H., 1995. Identification Manual for the Larval Chironomidae (Diptera) of Florida State of Florida. Florida State University, Tallahassee, pp: 110.

Fittkau, E.J. and S.S. Roback, 1983. The larvae of Tanypodinae (Diptera: Chironomidae) of the Holoarctic region-keys and diagnoses. Entomologica Scandinavica, 19: 33-110.

Henderson, P.A., 1990. Freshwater Ostracods: Keys and Notes for the Identification of the Species. Universal Book Services/W. Backhuys, Oegstgeest, The Netherlands, pp: 228.

Holthius, L.B., 1961. Report on a collection of crustacea and stamotopoda from Turkey and the Balkans, Zool. Verhandelungen No. 47, pp: 67.

Hynes, H.B.N., 1993. Adults and Nymphs of British Stoneflies (Plecoptera). Freshwater Biological Association, UK., pp: 90.

Karaman, G.S. and S. Pinkster, 1987. Freshwater Gammarus species from Europe, North Africa and Adjacent Regions of Asia (Crustacea-Amphipoda), Part III Gammarus balcanicus-group and related species. Bijdragen Tot de Dierkunde, 57: 207-260.

Kathman, R.D. and R.O. Brinkhurst, 1998. Guide to the Freshwater Oligochaetes of North America. Aquatic Resources Center, Tennessee, pp: 264.

Kazanci, N. and M. Dugel, 2000. An evaluation of the water quality of yuvarlakcay stream, in the koycegiz-dalyan protected area, SW Turkey. Turk. J. Zool., 24: 69-80.
Martens, K., 1997. Two new crenobiont ostracod genera (Crustacea, Ostracoda, Herpetocypridinae) fron Africa and Asia Minor, with the description of a new species from dolomitic springs in South Africa. South Afr. J. Sci., 93: 542-554.

Mozo, M.E.G., A. Baltanas and K. Martens, 1996. A Taxonomic Revision of European Herpetocypris (Brady and Norman, 1889 (Crustacea, Ostracoda)). Bulletin de L Institut Royal Des Sciences Naturelles de Belgique Biologie, 66: 93-132.

Nielsen, C.O. and B. Christensen, 1959. The Enchytraeidae, critical revision and taxonomy of European species. Natura Jutlandica, 8-9: 1-160.

Ozbek, M., M.R. Ustaoglu and S. Balik, 2003. Contribution to the knowledge on variability and ecology of Echinogammarus antalyae G. Karaman 1971 (Crustacea: Amphipoda: Gammaridae). Zool. Middle East, 29: 113-115.

Parker, S.P., 1982. Synopsis and Classification of Living Organizms. Vol. 2, McGraw-Hill, New York.

Pennak, R.W., 1953. Fresh-Water Invertebrates of the United States. The Ronald Press Company, New York, pp: 769.

Savage, A.A., 1989. Adult of the British Aquatic Hemiptera Heteroptera: A Key with Ecological Notes. Freshwater Biological Association, UK., pp: 173.

Sperber, C., 1950. A guide for the determination of European Naididae. Zool. Bidrag Uppsala Bd., 29: 45-78.

Timm, T., 1999. A Guide to the Estonian Annelida. Naturalist's Handbooks 1. Estonian Academic Publishers, Tartu-Tallinn, pp: 208.

Zhadin, V.I., 1952. Mollusks of freshwater and brackish water of the USSR and adjacent countries. Translated from Russian by Israel Program for Scientific Translation, Jerusalem, 1965, pp: 368. 